\title{
THREE-DIMENSIONAL POSTURE ESTIMATION OF FOOT BONES BY USING PLANTAR PLATE
}

\author{
Kenta Nomura*, , Teru Yonezawa*, Shinichi Kosugi ${ }^{\dagger}$, \\ Yasuhito Tanaka ${ }^{\ddagger}$, Hiroshi Mizoguchi* and Hiroshi Takemura* ${ }^{*}$ \\ *Department of Mechanical Engineering, Tokyo University of Science \\ 2641 Yamazaki, Noda-City, Chiba 278-8510, Japan \\ ${ }^{\dagger}$ Department of Orthopedic Surgery \\ Nara Prefectural Seiwa Medical Center, Nara, Japan \\ ${ }^{\ddagger}$ Department of Orthopedic Surgery \\ Nara Medical University, Nara, Japan \\ \$7515648@ed.tus.ac.jp \\ Ttakemura@rs.tus.ac.jp
}

Received 16 November 2016

Accepted 14 June 2017

Published 21 July 2017

\begin{abstract}
Purpose: This paper proposes a method to easily and quantitatively estimate the changes in the foot bone three-dimensional (3D) posture from the 3D posture of a plantar plate without using X-ray or computed tomography (CT). Methods: The estimation functions from the posture of the plantar plate attached to the sole of a foot to the posture of the each bone are calculated using multiple regression analysis (MRA). Because we assumed that the posture of the plantar plate is related to each bone posture. Each bone posture can be estimated by substituting the plantar plate posture into the estimation function. Results: The adjusted coefficient of determination of the linear regression model (estimation function) of more than $90 \%$ was obtained by the estimation function, which was higher than 0.70. The estimation accuracy root mean square error (RMSE) of the translation and rotation were approximately within $\pm 2.8 \mathrm{~mm}$ and $\pm 2.9^{\circ}$, respectively. The RMSE/range of motion (RoM) values of the translation and rotation were approximately within $\pm 22.1 \%$ and $\pm 7.1 \%$, respectively.
\end{abstract}

Correspondence to: Hiroshi Takemura, 2641 Yamazaki, Noda, Chiba 278-8510, Japan.

This is an Open Access article published by World Scientific Publishing Company. It is distributed under the terms of the Creative Commons Attribution 4.0 (CC-BY) License. Further distribution of this work is permitted, provided the original work is properly cited. 
Conclusion: The experimental results suggest that the 3D posture of almost all types of foot bones can be easily estimated using plantar plate posture and the linear regression model. This is an inexpensive, easy-to-apply method that can perform real-time measurement.

Keywords: Three-dimensional posture estimation; Ankle foot bones; Plantar plate; Globally optimal iterative closest point.

\section{INTRODUCTION}

Quantitative analysis of a three-dimensional (3D) joint motion is very important from the point of view of diagnosis and treatment support. Because the analysis results can be applied to support investigation of a joint surgical operation method, measurement of the range of motion (ROM) before and after operation, and in objective evaluation such as disease in a joint or a functional disorder. In particular, the ankle plays an important role in human walking, which is a basic human motion. Even a small decrease in the ankle joint ROM greatly affects the daily activities. An objective and quantitative ankle joint motion analysis is thus required. However, quantitative evaluation of an ankle joint motion with six degrees of freedom (6-DOF) is not easy because the ankle joint is a compound joint consisting of 26 bones as well as the hand. ${ }^{13}$ Therefore, in the field of biomechanics, great interest is extended on the motion measurement of bones that constitutes the ankle joint because many factors about the detailed and quantitative motion mechanism of ankle joints remain unclear. Three-dimensional quantitative ankle joint motion analyses can contribute to technical and scientific development in many fields such as surgery, rehabilitation, sports science, and biomechanics.

In previous studies in clinical fields, X-ray equipment, computed tomography (CT), magnetic resonance imaging (MRI), motion capture system (MCS), or 2D/3D registration has been used to measure bone posture or in motion analysis. X-ray fluoroscopy can perform consecutive photography and can measure dynamic changes in the bone. ${ }^{3,12} \mathrm{CT}$ and MRI can measure in three dimensions, and an operator can visually and intuitively understand the surface shape of the bone, internal structure, and alignment of joints. ${ }^{410}$ The MCS method, which measures the $3 \mathrm{D}$ position of the marker located on the skin using multiple cameras, can quantitatively evaluate the $3 \mathrm{D}$ posture of the foot. ${ }^{11}$ The $2 \mathrm{D} / 3 \mathrm{D}$ registration method matches the $3 \mathrm{D}$ model of the bone of a subject generated by CT or MRI beforehand in the transparent image obtained by $\mathrm{X}$ ray with a $2 \mathrm{D}$ image of the edge of a bone and calculates the $3 \mathrm{D}$ posture of the bone using a computer. ${ }^{1,2,8,19}$ However, all the above-described methods suffer from many problems such as the necessity of technical knowledge, preparation and analysis time for the operator, and limitation of the measurement location. Some previous studies were conducted that clarified the change in the foot bone posture or joint alignment using X-ray or CT data. ${ }^{4-7,9}$ However, no study was made that proposed an estimation method for the change in the foot bone posture that does not use X-ray, CT, or MRI. On the basis of the above background information and issues, the present study aims to easily and quantitatively estimate the postures of an foot bone with 6-DOF for application in the medical field. In our previous study, we developed a measurement device (PHARAD) that can measure the motion of a human foot joint with 6-DOF. ${ }^{17,18}$ We therefore propose an estimation method of the change in the foot bone posture from the posture of a plantar plate attached to the sole of a foot for application of our developed device. 


\section{METHOD}

\section{Estimation Method from the Plantar Plate}

In the present study, we assumed that the posture of the plantar plate is related to each bone posture. The estimation functions from the plantar plate posture to each bone posture are calculated using multiple regression analysis (MRA). Each bone posture can be estimated by substituting the plantar plate posture into the estimation function. Multiple bone posture and plantar plate posture data are needed to obtain the estimation function using MRA. Therefore, we conducted experiments to measure the plate and bone postures using CT equipment. Each bone posture and the plantar plate posture data were calculated using the slice image data of CT. We obtained the estimation function using MRA. In this study, we used the change in each bone posture from the initial bone posture $(\triangle$ PPlate and $\triangle$ PBone, as shown in Fig. 1) and not the absolute value of each bone posture for the calculation or estimation.

\section{Generation of the Estimation Function}

We used a linear regression model as an estimation function and used the MRA, which is one of the multiple classification analyses. In this method, one component of each bone posture is estimated using some components of the plantar plate posture. For example, if we perform MRA for translation $x$ of the bone posture, which is

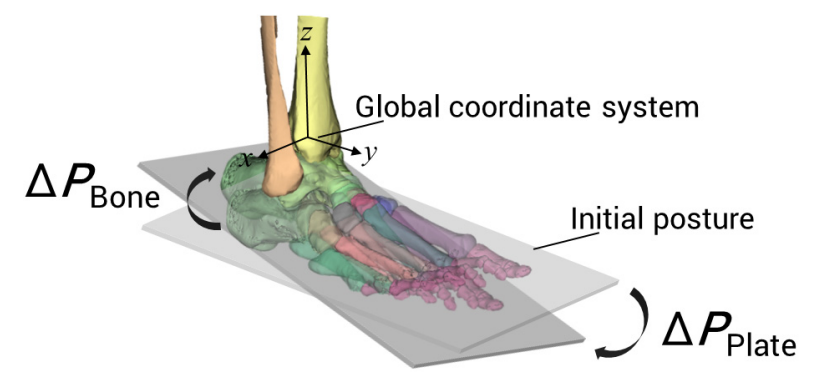

Fig. 1 Change in the plantar plate and foot bone posture. defined as a dependent variable, then a few of the six components (translations $x, y$, and $z$ and rotations $\theta, \varphi$, and $\psi$ ) of the plantar plate posture are defined as independent variables. Stepwise regression analysis was used to select the independent variables. For example, all components of the plantar plate posture were defined as independent variables; thus, we obtained the estimation function expressed by (1).

$$
x_{b}=a_{1} x_{p}+a_{2} y_{p}+a_{3} z_{p}+a_{4} \theta_{p}+a_{5} \varphi_{p}+a_{6} \psi_{p}+a_{7}
$$

Translations $y$ and $z$ and rotations $\theta, \varphi$, and $\psi$ of each bone posture can be estimated using the same procedure. In this study, $x, y$, and $z(\mathrm{~mm})$ represent the 3D positions in the directions of the $x-, y$-, and $z$-axes, respectively, and $\theta, \varphi$, and $\psi$ represent rotational angles $\theta, \varphi$, and $\psi\left(^{\circ}\right)$ around the $x-, y$-, and $z$-axes, respectively, as defined in Sec. 3.2. In other words, $\theta, \varphi$, and $\psi$ show the dorsi-plantar flexion (DF-PF), inversion-eversion (IN-EV), and adduction-abduction (AD$\mathrm{AB})$ directions, respectively.

\section{EXPERIMENT \\ CT Photography Experiment}

Six healthy adult men that do not have any disorder or surgical history in the lower limb were recruited, and five types of static postures of the right foot with the plantar plate were measured by CT (Aquillion64, Toshiba Medical Systems Co., Ltd.). The age, height, weight, foot length, and foot width were $31.2 \pm 10.2$ years, $169.9 \pm 1.9 \mathrm{~cm}, 54.6 \pm 2.2 \mathrm{~kg}, 24.2 \pm 0.6 \mathrm{~cm}$, and $9.4 \pm 0.6 \mathrm{~cm}$, respectively. The experiment was approved by the ethics committee of Nara Prefecture Western Medical Center and was conducted in full compliance with the Helsinki Declaration. Informed consent from the subjects was obtained. Figure 2 shows the experimental 


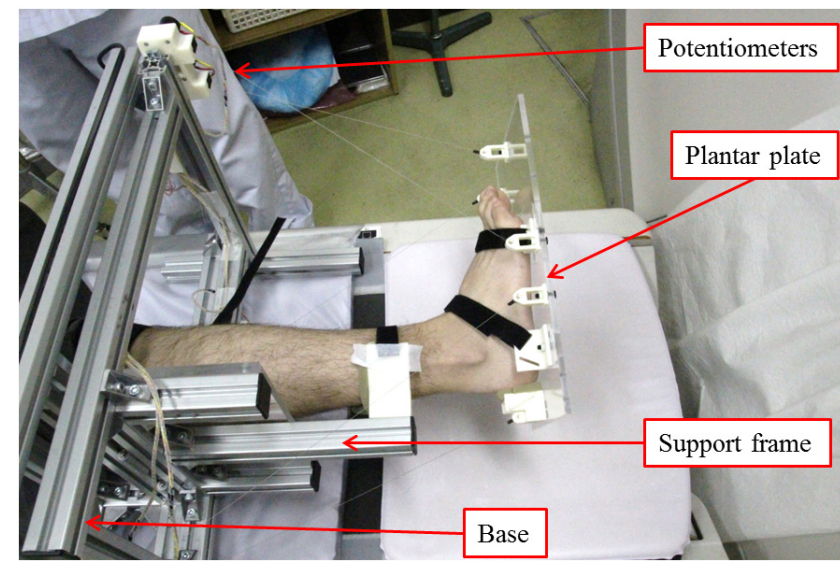

Fig. 2 Experimental foot posture measurement device.

device, which modified the shape of the 6-DOF motion-measuring device for the human foot developed in our previous research. This device can provide measurement of the foot posture with 6-DOF whose measurement is difficult using the conventional method. This device is composed of a base and a platform connected by six linear-wire potentiometers and can measure and calculate the foot posture with 6-DOF using the length of these potentiometers and an inverse kinematic method. The leg of the subject was prevented from moving during the photography process by fixing the lower limb using the support frame and belt of this device.

The plantar plate is made of an acrylic board so as not to affect the photography process using CT. For the details of the foot posture measurement method and the construction of this device, please refer to our previous study because this device is similar to the device used in our previous work except for the shape. ${ }^{17,18}$

The plantar plates were attached to the foot of the subjects in a left decubitus position and were photographed using CT. An initial posture (DF/ $\mathrm{PF}=0^{\circ}, \mathrm{IV} / \mathrm{EV}=0^{\circ}$, and $\mathrm{AD} / \mathrm{AB}=0^{\circ}$ ) and four combined postures of $\mathrm{DF} / \mathrm{PF}$ and $\mathrm{IV} / \mathrm{EV}$, i.e. $\mathrm{DF}+\mathrm{IV}, \mathrm{DF}+\mathrm{EV}, \mathrm{PF}+\mathrm{IV}$, and $\mathrm{PF}+\mathrm{EV}$, were evaluated, as shown in Fig. 3. The slice pitch of

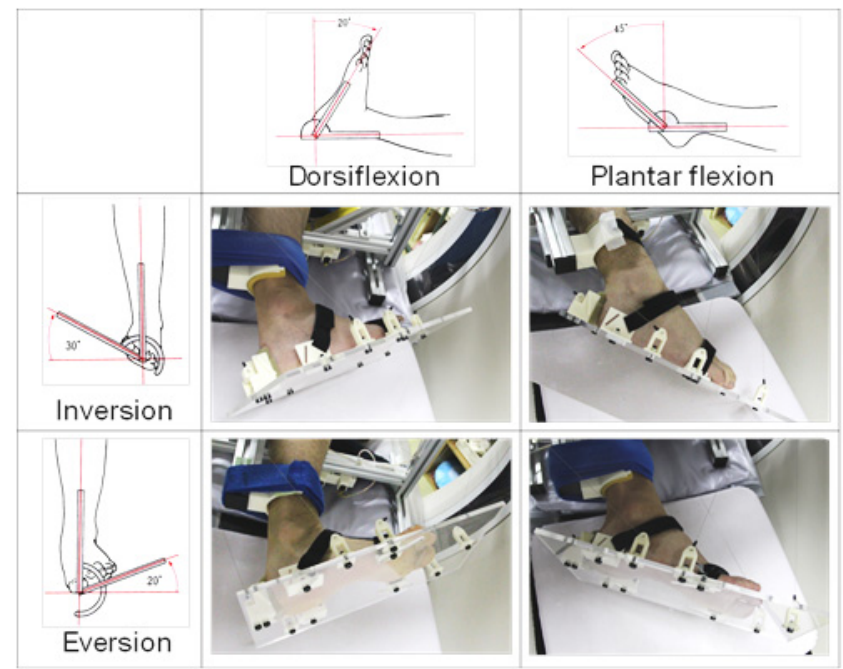

Fig. 3 Evaluated foot postures.

the CT was $0.5 \mathrm{~mm}$ and the photographing range was a cylindrical shape with a diameter of $400 \mathrm{~mm}$, and in the axial direction, the lower part of the tibia (about $150 \mathrm{~mm}$ from the medial malleolus to the knee side) was taken as the imaging range from the toe. In addition, we instructed all subjects to continue setting all their fingers to the plantar plate and to maintain foot posture during photography. Only three types of posture were photographed in one experiment to avoid excessive radiation exposure. In the first experiment, the initial postures, DF + IV, and PF + EV were photographed. In the second experiment, the initial postures, $\mathrm{DF}+\mathrm{EV}$, and $\mathrm{PF}+\mathrm{IV}$ were photographed. The interval between the two experiments was approximately one-half year.

\section{Data Analysis}

The data obtained by CT are mostly radiation transmission images. The images are outputted in the form of medical image standard called Digital Imaging and COmmunication in Medicine (DICOM). In this experiment, the DICOM and concentration resolution values were $512 \times 512$ pixels and 16 bits, respectively. 
First, a 3D model of each bone was generated from DICOM using VoTracer (Open source, RIKEN). By arbitrarily setting the CT number range, it is possible to display only specific tissues such as bones and soft tissues. In this study, the minimum and maximum CT number were set to 100 and 1000, respectively, so that bone areas could be clearly observed in all subjects. The change in posture with 6-DOF of each bone from the initial posture in the global coordinate system was calculated. Second, the tibia at the initial posture was defined as the global coordinate system. Third, the change in the posture with 6DOF of each bone from the initial posture in the global coordinate system was calculated after the tibia in the four combined postures was matched with the bone at the initial posture. For the 3D image visualization, the area division software "VoTracer" developed by RIKEN was used to generate a 3D model of each bone. VoTracer can three-dimensionally extract the area chosen by an operator and output the chosen area in 3D model format (Stereolithograpy, STL). Region growing, which is one of the functions of VoTracer, was used to extract the area. We obtained the point group data to calculate the change in the bone posture after extracting each bone area and generating the 3D bone model. Figure 4 shows the definition of the global coordinate system of the tibia. The lateral malleolus, second-toe, and tibialaxis directions from the origin are defined as the $x-, y-$, and $z$-axes of the global coordinate system, respectively. Figure 4(a) shows the definition of the origin, $x$-, and $y$-axis directions. The bottom of the tibia was made to approximate a square. The intersection point of the perpendiculars of two isosceles triangles whose extended two sides faced each other was set as the origin. In addition, the perpendicular line of the second-toe direction was defined as the $y$-axis direction, and the axis perpendicular to the $y$-axis direction was defined as the $x$-axis direction. However, in this state, the

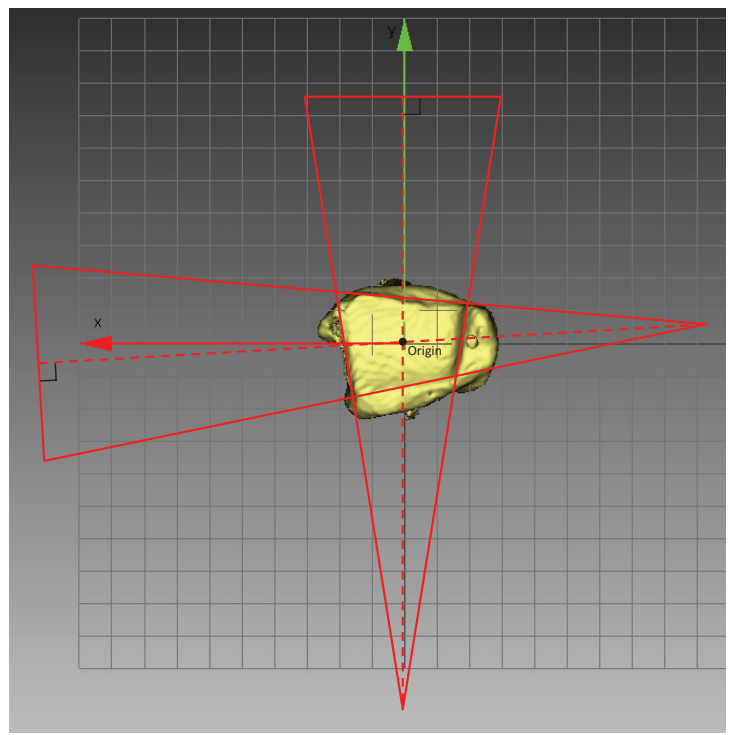

(a)

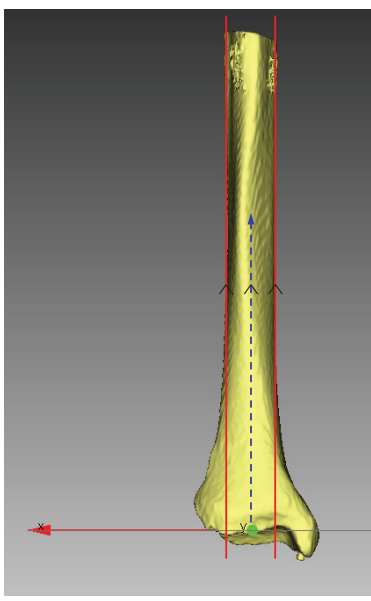

(b)

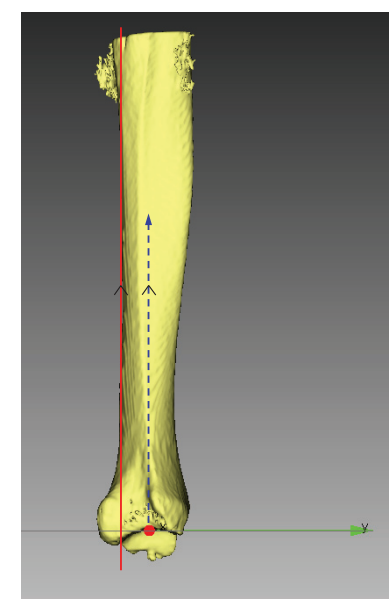

(c)
Fig. 4 Definition of the global coordinate system set of the tibia. (a) Definition of $x$ - and $y$-axis, (b) Definition of $x$ - and $z$-axis and (c) Definition of $y$ - and $z$-axis.

$x$-axis is around the $y$-axis, the $y$-axis is around the $x$-axis, the $z$-axis has DoF about both $x$ and $y$ axes, and the coordinate system cannot be uniquely determined. Therefore, as shown in Figs. 5(b) and 5(c), the rectilinear contour of the tibia is linearly approximated, and the orthogonal coordinate system is uniquely determined by determining the direction of the $z$ axis so as to be parallel to the approximated straight line. 


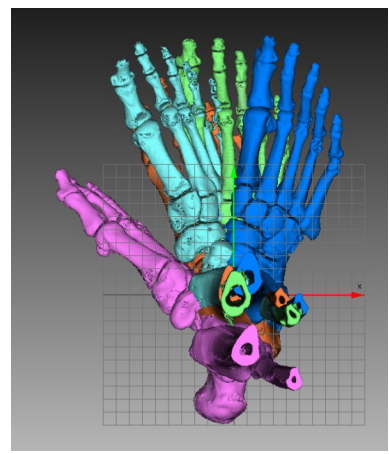

(a)

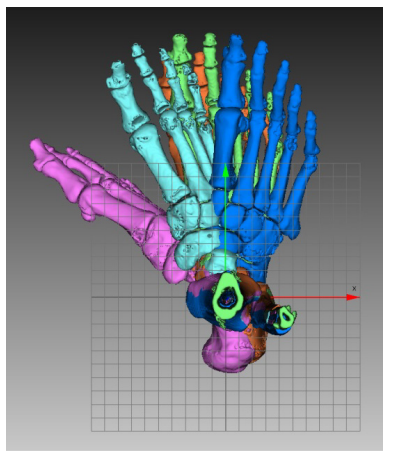

(b)
Fig. 5 3D model of each foot posture. (a) Before tibia fitting and (b) After tibia fitting.

In this manner, the global coordinate system was uniquely determined. Each 3D model of the five types of posture obtained using CT had different positions, as shown in Fig. 5(a). Therefore, we need to set a global coordinate to the tibia of each data when we defined the tibia in a global coordinate system. The four coordinate systems of the tibia in the four combined postures must be matched at the initial posture, as shown in Fig. 5(b). In this study, the globally optimal iterative closest point (Go-ICP) algorithm was used for objective and highly accurate matching of each coordinate system. ${ }^{15,16}$ The Go-ICP algorithm is one of the automated 3D registration methods, which is composed of the branch and bound and voxel ICP and can efficiently derive an optimal solution. By using the Go-ICP algorithm, reliable calculated data with high accuracy can be automatically obtained. In addition, the change in each bone posture from the initial bone posture was automatically calculated using GoICP. The change in each bone posture was calculated using a homogeneous transformation matrix, which was used to match the point group of the initial bone posture with that of each bone posture calculated by Go-ICP. The second or third cuneiform experienced difficulty in individually converging when each bone posture was calculated. When the shape of the point group does not have a specific characteristic, the result of the Go-ICP calculation sometimes does not converge. Therefore, we combined the two pointgroup data, which individually hardly move, into one point group (the combination of the second and third cuneiform is defined as one type of bone) to provide a characteristic to the point group data. After this combination, the second and third cuneiform postures can be calculated with high accuracy using Go-ICP. In this study, a cross-validation method was used to evaluate the estimation accuracy of the bone posture from the plantar plate. The training data consisted of five of the six plantar plates and the bone posture data obtained by the experiments used for the MRA as well as the test data of the remaining data that were not used for MRA [Fig. 6(1)]. The bone posture of the test data was estimated by the plate posture of the test data from the estimation function [Fig. 6(2)]. The error was calculated by comparing the estimated and true values using Go-ICP [Fig. 6(3)]. We changed the combination of the training data for the MRA, estimated the test data of all subjects, and calculated the error of all patterns. Subsequently, the root mean square error (RMSE) was calculated from all the errors to evaluate the estimation accuracy of this method.

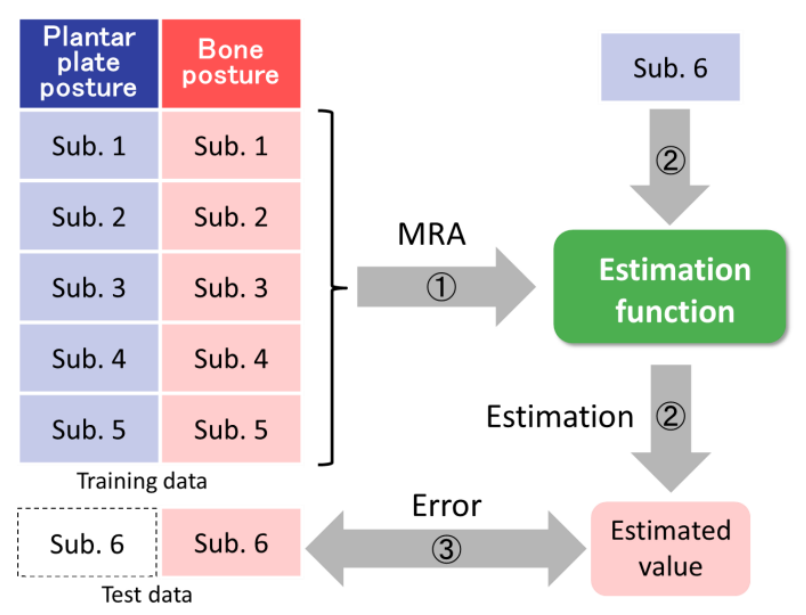

Fig. 6 Evaluation method of the bone posture estimation. 
Three-Dimensional Posture Estimation of Foot Bones by Using Plantar Plate

Table 1 Adjusted R2 of the Regression Equation in the Tibia Coordinate System (Bold: $R 2>0.70)$.

\begin{tabular}{|c|c|c|c|c|c|c|c|c|c|c|c|c|}
\hline & & & & & 1st & 2nd & 3rd & 1st & 2nd & 3rd & 4th & 5th \\
\hline & Talus & Calcaneus & Cuboid & Navicular & Cuneiform & Cuneiform & Cuneiform & Metatarsal & Metatarsal & Metatarsal & Metatarsal & Metatarsal \\
\hline$x$ & 0.22 & 0.66 & 0.82 & 0.86 & 0.91 & 0.88 & 0.88 & 0.9 & 0.77 & 0.84 & 0.9 & 0.9 \\
\hline$y$ & 0.96 & 0.92 & 0.9 & 0.87 & 0.81 & 0.85 & 0.85 & 0.88 & 0.9 & 0.73 & 0.89 & 0.9 \\
\hline$z$ & 0.33 & 0.75 & 0.9 & 0.85 & 0.83 & 0.86 & 0.86 & 0.85 & 0.57 & 0.49 & 0.77 & 0.84 \\
\hline$\theta$ & 0.98 & 0.99 & 0.99 & 0.99 & 0.99 & 0.99 & 0.99 & 0.99 & 0.98 & 0.99 & 0.99 & 0.99 \\
\hline$\varphi$ & 0.27 & 0.97 & 0.97 & 0.97 & 0.98 & 0.98 & 0.98 & 0.98 & 0.97 & 0.95 & 0.98 & 0.98 \\
\hline$\psi$ & 0.83 & 0.97 & 0.98 & 0.98 & 0.98 & 0.98 & 0.98 & 0.98 & 0.98 & 0.97 & 0.99 & 0.99 \\
\hline
\end{tabular}

\section{RESULT}

\section{R2 of the Estimation Function}

Table 1 lists the adjusted coefficient of determination (R2) of the linear regression model

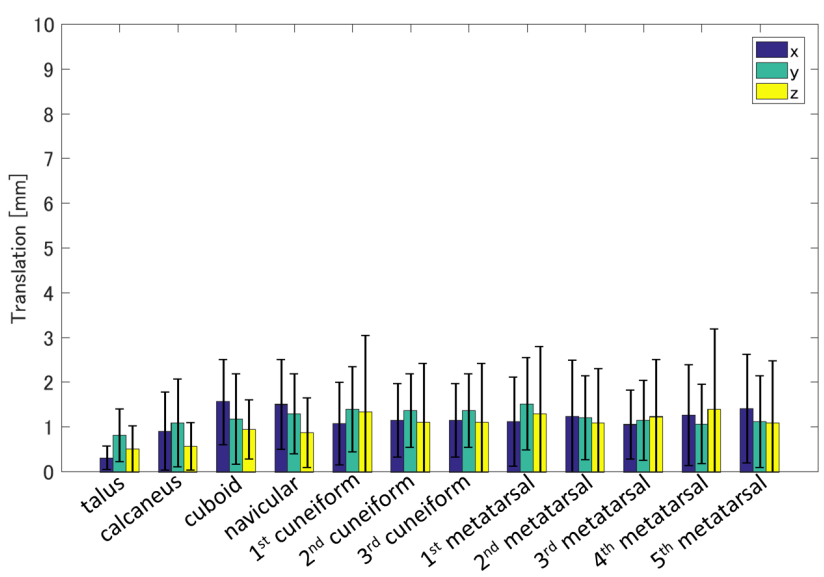

(a)

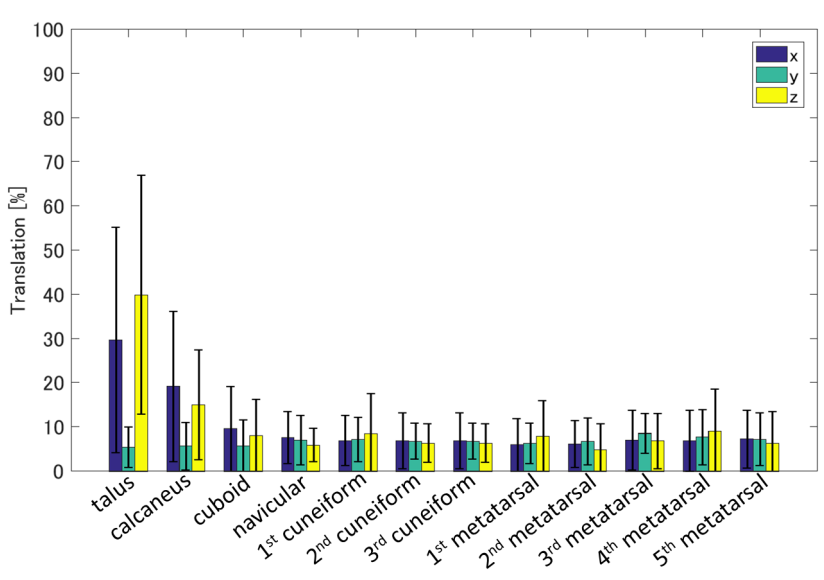

(c) (estimation function) of each bone posture component in the four combined postures. In this study, we defined $R 2>0.7$ as highly reliable. The bold font in Table 1 shows $R 2>0.7$. In Table 1 , $\mathrm{R} 2$ of the rotation estimation function, except $\varphi$ of

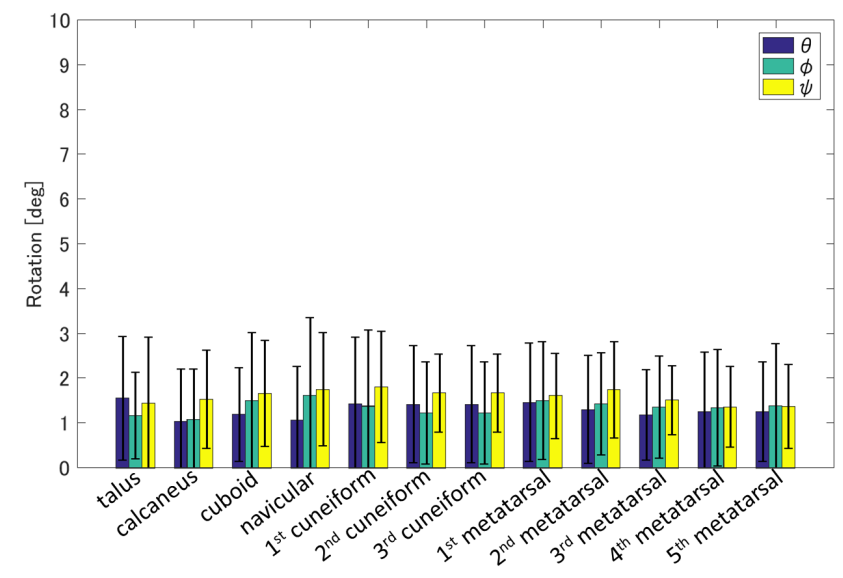

(b)

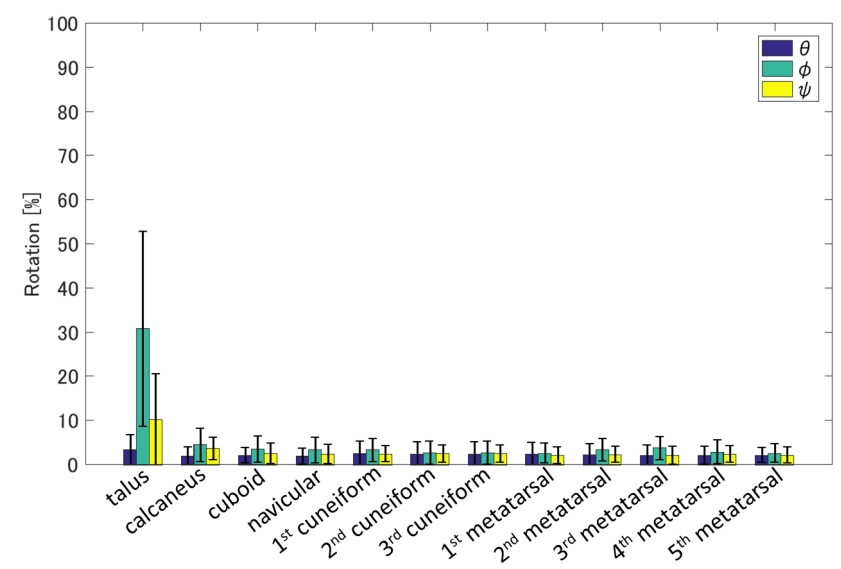

(d)

Fig. 7 Posture estimation RMSE of each bone. (a) RMSE in the translation direction, (b) RMSE in the rotation direction, (c) RMSE/ROM in the translation direction and (d) RMSE/ROM in the rotation direction. 
the talus, was higher than 0.70 and almost higher than 0.90 . On the other hand, R2 of the translation estimation function was almost higher than 0.70; however, those of the talus and second and third metatarsal were lower than 0.70 . In all bone types, $\mathrm{R} 2$ of the rotation estimation function tended to be higher than that of the translation estimation function. Figure 7 shows the posture estimation accuracy of each bone. Figures 7(a)-7(d) show the RMSE of the translation, RMSE of the rotation, percentage of RMSE to ROM (mean ROM of each bone of the subject, RMSE/ROM) of the translation, and RMSE/ROM of the rotation, respectively. The error bars represent the standard deviations of each RMSE for all subjects. Figures $7(a)$ and $7(b)$ show the estimated RMSEs of the translation and rotation, which are approximately within $\pm 2.8 \mathrm{~mm}$ and $\pm 2.9^{\circ}$, respectively. Figures 7(c) and 7(d) show the estimated RMSE/ROM of the translation and rotation except for the talus, which were approximately within $\pm 22.1 \%$ and $\pm 7.1 \%$, respectively. The RMSE/ROM values of $x, z$, and $\varphi$ of the talus, $x$ of the calcaneus, and the second metatarsal, which have low R2, showed a tendency higher than that of the other bones. This tendency was particularly visible in the talus.

\section{DISCUSSION}

In this study, the change in the bone posture was estimated from a plantar plate posture using the linear regression model expressed as (1) in Sec. 2.2. As a result, the R2 value of more than $90 \%$ of the estimation function obtained in Sec. 2.2 was higher than 0.70 , as listed in Table 1. This result suggests that the posture of almost all types of foot bones can be explained using a linear regression model obtained from the plantar plate posture. The cause of low R2 of the $\varphi$ estimation function for the talus was the influence of the talocrural joint motion. The talus does not move when the IV-EV motion of the plantar plate occurs only at the subtalar joint.

However, the IV-EV motion of the plantar plate occurs at the talocrural joint. The ROM of the talocrural joint is determined by the shape of the tibia and fibula and is different depending on the individual characteristic. In other words, the IV-EV motion of the talus is different according to the individual characteristic and cannot be explained by the linear regression model. This factor contributed to this result. R2 of the $x$ and $z$ estimation function of the talus realized a low value for similar reason. On the other hand, the results show that the other motion of the talus can be explained by the linear regression model. The motion of the talus at the talocrural joint is mainly a DF-PF motion. Therefore, the ratio of the motion difference based on individual characteristic is low. The difference based on the individual characteristic did not occur because the $\mathrm{AD}-\mathrm{AB}$ motion of the talus at the talocrural joint hardly moved. These two factors contributed to this result. In addition, R2 of the rotation estimation function, except the talus, was higher than 0.95 . This result suggests that the motion of the bones near the plantar plate is related to the motion of the plantar plate and can be explained by the linear regression model. R2 of the translation estimation function of the metatarsal was lower than that of the other bones because the difference based on individual characteristic affected the translation of the metatarsal. In this study, the center of the bone mass was defined as the origin and was used to calculate the change in the translation. The center of the bone mass differed according to the foot length or foot width because it was determined from the bone shape. The difference in the center of the bone mass was found to be remarkably bigger than that of the other types of bones because the metatarsal is a long and narrow bone. Therefore, the R2 value of the translation estimation function of the 
metatarsal was low. The R2 value of the estimation function of the translation was lower than that of the rotation in all bones because the factors have difficulty estimating the change in the plantar plate posture caused by the different bone shapes. With regard to the estimation accuracy caused by the individual differences, the reliability of the estimation function can be increased by finding a correction parameter. However, the correction parameter must be determined from a parameter that can be easily measured, e.g. foot length, foot width, or ROM of the plantar plate, because the benefits of this method come from not using X-ray, CT, or MRI.

Investigation of the correction parameter, derivation of the estimation function of each posture, and examination of the nonlinear regression model were not performed in this study. These will be investigated in the future by finding a higher reliability regression model.

This result cannot present a conclusion whether this accuracy is sufficient or not because the required estimation accuracy cannot be determined for a useful purpose. When a useful purpose for a surgical operation method can be obtained, an objective evaluation before or after operation would require accuracy of less than $1 \mathrm{~mm}$ and $1^{\circ}$. This method is unsuitable for use under these conditions because the accuracy obtained by this method cannot satisfy these demands. Because the joint angle is expressed in $5^{\circ}$ steps in the rehabilitation field, the accuracy obtained in the present experiment can be considered to lie within the allowable range. In other words, this method can be used for measurement of ROM before and after rehabilitation or evaluation, such as disease in a joint or functional disorder. With regard to the RMSE/ROM except the talus that of the translation is almost $10 \%$ and that of the rotation is almost $5 \%$. The estimation accuracy of this method is not low when we consider that the estimation did not use X-ray and CT. With regard to the RMSE/ROM of the talus, because the error in the $\theta$ rotation direction, which is the largest ROM of the ankle joint motion during gait, is very small, i.e. $4.4 \%$, the talus posture in the DF-PF direction can be estimated using this method. However, the RMSE/ROM value of the bone posture, whose $\mathrm{R} 2$ is low, is high. This result suggests that estimating the talocrural joint motion and translation of the metatarsal, whose individual differences are large compared with the plantar plate posture, is difficult. The most highly accurate and mainstream method is the $2 \mathrm{D} / 3 \mathrm{D}$ registration by objectively performing $3 \mathrm{D}$ change measurement of each bone posture. This section compares the advantage and disadvantage of this proposed method with those of the $2 \mathrm{D} / 3 \mathrm{D}$ registration and discusses the benefits and role of the proposed method. According to a previous study on 2D/3D registration by Yamazaki et al., the accuracy of the RMSE values of the translation and rotation was approximately within $\pm 1.5 \mathrm{smm}$ and $\pm 0.8^{\circ}$ of the 2D/3D registration method, respectively. ${ }^{14}$ This method satisfies the required accuracy in surgery (within $1 \mathrm{~mm}$ and $1^{\circ}$ ) and has a high accuracy in measuring bone postures. ${ }^{14}$ On the other hand, because the proposed method produces errors of almost $5 \mathrm{~mm}$ and $5^{\circ}$, the $2 \mathrm{D} / 3 \mathrm{D}$ registration should be used for accurate measurement. However, our proposed method is not time consuming, in contrast to the $2 \mathrm{D} / 3 \mathrm{D}$ registration, because this proposed method can estimate the bone posture using only the plantar plate posture with 6-DOF and can provide the result for substitution of the linear regression model. In addition, all bone postures and foot alignment can be instantly displayed using this method because PHARAD can measure postures with 6-DOF of the plantar plate in real time. In addition, this method does not require special environment or complicated preparations because PHARAD, which is a portable device, is used to measure 
the plantar plate posture. Therefore, a doctor or physical therapist that does not have the technical knowledge can perform simple inspection and detect the disease of the joint at its early stages.

PHARAD can control the 3D foot bone posture to provide effective rehabilitation using this method because the relationship of the plantar plate posture and each bone posture is clarified in this study. From these results, the proposed method is useful from the viewpoint of convenience, is not time consuming, and can be applied in real time.

\section{LIMITATION}

In this study, we experienced difficulty in stating that the many data generated for MRA are sufficient (six subjects and four types of posture). The number of subjects and postures may be increased to obtain higher reliability and accuracy. The applied regression model is an estimation function for bones with normal shapes because all subjects were healthy adult men. Therefore, measurement of the bone posture may be difficult using the proposed method when the bone shape is significantly different from the bones of healthy adult men, such as the bones of women, bones of children, artificial bones, or bones of arthrosis patients.

Furthermore, the active posture of the subject was photographed in this experiment under no load. The estimation error may be large under an external load because the posture of the foot bone will be affected by an external load. Therefore, the proposed method is limited to posture estimation of bones when the subjects assume an active posture without load. However, the foot bone posture under a load can be estimated by photographing the foot bone using CT, and generation of the regression model can be estimated because this experiment protocol can measure the foot bone posture with attached plantar plate under load. With regard to the regression model, the linear regression model was adopted in this study. However, it is insufficient in estimating a target bone or posture. In addition, the same regression model was used for all subjects, and investigation of individual differences in the posture of the bone has not been performed yet. Therefore, the regression model of this method is insufficient in estimating the bone posture, which has a large individual difference, and introducing some types of correction parameters is necessary.

\section{CONCLUSION}

Because the objective of this study is the development of a method to easily and quantitatively estimate postures of the foot bone with 6-DOF without using X-ray or $\mathrm{CT}$, we have proposed a method that can calculate the changes in the foot bone posture from the posture of a plantar plate attached to the sole of a foot. The proposed method maintains that each bone posture can be estimated by substituting the plantar plate posture using an estimation function (linear regression model) calculated by MRA. R2 was calculated to evaluate whether the linear regression model can explain the posture of almost all types of foot bones. As a result, R2 of more than $90 \%$ was obtained by the estimation function, which was higher than 0.70 . This result suggests that the posture of almost all types of foot bones can be explained using the linear regression model obtained from the plantar plate posture. RMSE and RMSE/ROM were calculated from all the errors in the true and estimated values to evaluate the estimation accuracy of the proposed method. The RMSE values of the translation and rotation were approximately within $\pm 2.8 \mathrm{~mm}$ and $\pm 2.9^{\circ}$, respectively, and the RMSE/ROM values of the translation and rotation, except for 
the talus, were approximately within $\pm 22.1 \%$ and $\pm 7.1 \%$, respectively. The experimental results suggest that the 3D bone posture can be easily estimated without using X-ray or CT. In the future, we will develop a rehabilitation device to control 3D foot bone postures to provide effective rehabilitation using the proposed method.

\section{ACKNOWLEDGMENTS}

We represent that this submission is original work, and is not under consideration for publication with any other journal.

\section{References}

1. Akiyama K, Noh B, Fukano M, Miyakawa S, Hirose N, Fukubayashi T. Analysis of the talocrural and subtalar joint motions in patients with medial tibial stress syndrome. J Foot Ankle Res 8(25): 2015.

2. Balsdon MER. In-vivo investigation of the medial longitudinal arch of the foot and orthotic interactions using bi-planar fluoroscopy. Electronic Thesis and Dissertation Repository Paper 570. Thesis, The University of Western Ontario, Canada, 2012.

3. Coetzee JC, Castro MD. Accurate measurement of ankle range of motion after total ankle arthroplasty. Clin Orthop Relat Res 424: 27-31, 2004.

4. de Asla RJ, Wan L, Rubash HE, Li G. Six dof in vivo kinematics of the ankle joint complex: Application of a combined dual orthogonal fluoroscopic and magnetic resonance imaging technique. J Orthop Res 24(5): 10191027, 2006.

5. Goto A, Morimoto H, Itohara T, Watanabe T, Sugamoto $\mathrm{K}$. Three-dimensional in vivo kinematics of the subtalar joint during dorsi-plantarflexion and inversion-eversion. Foot Ankle Int 30(2): 432-438, 2009.

6. Gutekunst DJ, Liu L, Ju T, Prior FW, Sinacore DR. Reliability of clinically relevant $3 \mathrm{D}$ foot bone angles from quantitative computed tomography. J Foot Ankle Res 6(1): 2013.

7. Imai K, Ikoma K, Maki M, Kido M, Tsuji Y, Takatori R, Tokunaga D, Inoue N, Kubo T. Features of hindfoot 3D kinetics in flat foot in ankle-joint maximal dorsiflexion and plantarflexion. J Orthop Sci 16(5): 638-643, 2011.
8. Ito K, Hosoda K, Shimizu M, Ikemoto S, Kume S, Nagura T, Imanishi N, Aiso S, Jinzaki M, Ogihara N. Direct assessment of 3D foot bone kinematics using biplanar X-ray fluoroscopy and an automatic model registration method. J Foot Ankle Res 8(21): 2015.

9. Ledoux WR, Rohr ES, Ching RP, Sangeorzan BJ. Effect of foot shape on the three-dimensional position of foot bones. J Orthop Res 24(12): 2176-2186, 2006.

10. Liu G, Gao J, Yue H, Zhang X, Lu G. Design and kinematics simulation of parallel robots for ankle rehabilitation. Conf Mechatronics and Automation, pp. 11091113, 2006.

11. Mok KM, Fong DT, Krosshaug T, Hung AS, Yung PS, Chan KM. An ankle joint model-based image-matching motion analysis technique. Gait Posture 34(1): 71-75, 2011.

12. Schepers T, Ginai AZ, Mulder PG, Patka P. Radiographic evaluation of calcaneal fractures: To measure or not to measure. Skeletal Radiol 36(9): 847-852, 2007.

13. Schunke M, Schulte E. Thieme atlas of anatomy: General anatomy and musculoskeletal system. 2nd ed., IGAKUSHOIN, pp. 417-419, 2011.

14. Yamazaki T, Watanabe T, Nakajima $Y$, Sugamoto $K$, Tomita T, Maeda D, Sato Y, Yoshikawa H, Tamura S. Development of three-dimensional kinematic analysis system for artificial knee implants using $X$-ray fluoroscopic imaging. Japan Soc Radiol Technol 61(1): 79-87, 2005.

15. Yang J, Li H, Campbell D, Jia Y. Go-ICP: A Globally optimal solution to 3D ICP point-set registration. Trans Pattern Anal Mach Intell , 2016 (In press).

16. Yang J, Li H, Jia Y. Go-ICP: Solving 3D Registration efficiently and globally optimally. IEEE Int Conf Computer Vision, pp. 1457-1464, 2013.

17. Yonezawa T, Nomura K, Onodera T, Ding M, Mizoguchi $\mathrm{H}$, Takemura $\mathrm{H}$. Development and performance evaluation of parallel link type human ankle rehabilitation assistive device. IEEE Int Conf Robotics and Biomimetics, pp. 802-807, 2014.

18. Yonezawa T, Onodera T, Ding M, Mizoguchi H, Takemura H, Ogitsu, T. Development of three-dimensional motion measuring device for the human ankle joint by using parallel link mechanism. 36th Annual Int Conf IEEE Engineering in Medicine and Biology Society, pp. 4358-4361, 2014.

19. Zuffi S, Leardini A, Catani F, Fantozzi S, Cappello A. A model-based method for the reconstruction of total knee replacement kinematics. IEEE Trans Med Imaging 18(10): 981-991, 1999. 